\title{
An Independence Property for General Information
}

\author{
Doretta Vivona, Maria Divari \\ Department of Basic and Applied Sciences for Engineering, Faculty of Civil and Industrial Engineering, \\ "Sapienza"-University of Rome, Roma, Italy \\ Email: doretta.vivona@sbai.uniroma1.it, ID: 0000-0002-5267-6497,maria.divari@alice.it
}

Received 25 December 2015; accepted 19 February 2016; published 22 February 2016

Copyright (C) 2016 by authors and Scientific Research Publishing Inc.

This work is licensed under the Creative Commons Attribution International License (CC BY). http://creativecommons.org/licenses/by/4.0/

(c) (i) Open Access

\section{Abstract \\ The aim of this paper was a generalization of independence property proposed by J. Kampé de Fe- riét and B. Forte in Information Theory without probability, called general information. Therefore, its application to fuzzy sets has been presented.}

\section{Keywords}

\section{Information, Functional Equations, Fuzzy Sets}

\section{Introduction}

Since 1967-69, J. Kampé de Ferét and B. Forte have introduced, by axiomatic way, new information measures without probability [1]-[3]; later, in analogous way, with P. Benvenuti we have defined information measures without probability or fuzzy measure [4] for fuzzy sets [5] [6]. This form of information measure is again called general information.

In Information Theory an important role has played by an independence property with respect to a given information measures $J$ applied to crisp sets [7]. These sets are called $J$-independent (i.e. independent each other with the respect to $J$ ) [8].

For this reason we will propose a generalization of $J$-independence property.

The paper develops in the following way: in Section 2 we recall some preliminaires; in Section 3 the generalization of $J$-indepedence is proposed; the result is extended to fuzzy sets in Section 4. Section 5 is devoted to the conclusion.

\section{Preliminaires}

Let $\Omega$ be an abstract space and $\mathcal{C}$ the $\sigma$-algebra of crisp sets $C \subset \Omega$, such that $(\Omega, \mathcal{C})$ is a measurable 
space. We refer to [7] for all knoledge and operations among crisp sets.

J. Kampé de Ferét and B. Forte gave the following definition [1] [2]:

Definition 2.1 Measure of general information $J$ for crisp sets is a mapping

$$
J(\cdot): \mathcal{C} \rightarrow[0,+\infty]
$$

such that $\forall C, C^{\prime}, C_{1}, C_{2} \in \mathcal{C}$ :

(i) $C \subset C^{\prime} \Rightarrow J(C) \geq J\left(C^{\prime}\right)$,

(ii) $J(\varnothing)=+\infty, J(\Omega)=0$;

(iii) $J\left(C_{1} \cap C_{2}\right)=J\left(C_{1}\right)+J\left(C_{2}\right)$, if $C_{1} \cap C_{2} \neq \varnothing$.

If the couple $\left(C_{1}, C_{2}\right)$ satisfies the (iii), we say that $C_{1}$ and $C_{2}$ are $J$-independent, i.e. independent each other with respect to information $J$.

\section{A Generalization of the J-Independence Property}

In this paragraph we are going to present a generalization of the $J$-independence property.

We propose the following:

Definition 3.1 Given a general information $J$, let $C_{1}$ and $C_{2}$ be two crisp sets in $C$ such that $C_{1} \cap C_{2} \neq \varnothing$.

We say that $C_{1}$ and $C_{2}$ are J-idependent each other if there exists a continuous function $\Phi:[0,+\infty]^{2} \rightarrow[0,+\infty]$ such that

$$
J\left(C_{1} \cap C_{2}\right)=\Phi\left(J\left(C_{1}\right), J\left(C_{2}\right)\right)
$$

We shall characterize the function $\Phi$, taking into account the properties of the intersection for every $C_{1}, C_{2}, C_{3}, C_{1}^{\prime} \in \mathcal{C}$ :

$$
\left\{\begin{array}{l}
\left(p_{1}\right) \Phi\left(J\left(C_{1}\right), J\left(C_{2}\right)\right)=\Phi\left(J\left(C_{2}\right), J\left(C_{1}\right)\right), \text { commutativity } \\
\left(p_{2}\right) \Phi\left(\left(J\left(C_{1}\right), J\left(C_{2}\right)\right), J\left(C_{3}\right)\right)=\Phi\left(J\left(C_{1}\right),\left(J\left(C_{2}\right), J\left(C_{3}\right)\right)\right), \\
\quad \text { if } C_{1} \cap C_{2} \cap C_{3} \neq \varnothing \text { associativity } \\
\left(p_{3}\right) \Phi(J(C), J(\Omega))=J(C), \text { neutral element } \\
\left(p_{4}\right) C_{1} \subset C_{1}^{\prime} \Rightarrow \Phi\left(J\left(C_{1}\right), J\left(C_{2}\right)\right) \geq \Phi\left(J\left(C_{1}^{\prime}\right), J\left(C_{2}\right)\right), \\
\quad \text { if } C_{1}^{\prime} \cap C_{2} \neq \varnothing \text { monotonicity } \\
\left(p_{5}\right) \Phi\left(J\left(C_{1}\right), J\left(C_{2}\right)\right) \geq \bigvee\left(J\left(C_{1}\right), J\left(C_{2}\right)\right) .
\end{array}\right.
$$

Putting $J\left(C_{1}\right)=x, J\left(C_{2}\right)=y, J\left(C_{3}\right)=z, J\left(C_{1}^{\prime}\right)=x^{\prime}$, the properties $\left[\left(p_{1}\right)-\left(p_{5}\right)\right]$ have translated in the following system of functional equations and inequalities [9] [10]:

$$
\left\{\begin{array}{l}
\left(P_{1}\right) \Phi(x, y)=\Phi(y, x) \\
\left(P_{2}\right) \Phi(\Phi(x, y), z)=\Phi(x, \Phi(y, z)) \\
\left(P_{3}\right) \Phi(x, 0)=x \\
\left(P_{4}\right) x \geq x^{\prime} \Rightarrow \Phi(x, y) \geq \Phi\left(x^{\prime}, y\right) \\
\left(P_{5}\right) \Phi(x, y) \geq \bigvee(x, y) .
\end{array}\right.
$$

We can give the following

Proposition 3.2 A class of solutions of the system $\left[\left(P_{1}\right)-\left(P_{5}\right)\right]$ is

$$
\Phi_{h}(x, y)=h^{-1}(h(x)+h(y))
$$

where $h$ is any continuous, strictly increasing function $h:[0,+\infty] \rightarrow[0,+\infty]$ with $h(0)=0$ and 
$h(+\infty)=+\infty$.

Proof. The class of functions (2) satisfy the equations $\left[\left(P_{1}\right)-\left(P_{3}\right)\right]$ and the inequality $\left(P_{4}\right)$ by appling the Ling Theorem about the representation of a function which is monotone, commutative, associative with neutral element [11]. The inequality $\left(P_{5}\right)$ is a consequence of the monotonicity of $h$.

So, from (2), we have

Proposition 3.3 The generalization of the J-independence property for crisp sets is

$$
J\left(C_{1} \cap C_{2}\right)=h^{-1}\left(h\left(J\left(C_{1}\right)\right)+h\left(J\left(C_{2}\right)\right)\right), \forall C_{1}, C_{2} \in \mathcal{C}, C_{1} \cap C_{2} \neq \varnothing,
$$

where $h$ is any continuous, strictly increasing function $h:[0,+\infty] \rightarrow[0,+\infty]$ with $h(0)=0$ and $h(+\infty)=+\infty$.

Remark When $h$ is linear, the generalization (3) coincide with the property (iii).

\section{Extension to Fuzzy Setting}

In this paragraph, we are considering the extension of $J$-independence property at fuzzy setting.

Let $\Omega$ be an abstract space and $\mathcal{F}$ the $\sigma$-algebra of fuzzy sets such that $(\Omega, \mathcal{F})$ is a measurable space [5], [6]. In [4] we have given the definition of measure of general information for fuzzy sets:

Definition 4.1 Measure of general information in fuzzy setting is a mapping $J^{\prime}(\cdot): F \rightarrow[0,+\infty]$ such that $\forall F, F^{\prime}, F_{1}, F_{2} \in \mathcal{F}$ :

(i') $F \subset F^{\prime} \Rightarrow J^{\prime}(F) \geq J^{\prime}\left(F^{\prime}\right)$,

(ii') $J^{\prime}(\varnothing)=+\infty, J^{\prime}(\Omega)=0$,

(iii') $J^{\prime}\left(F_{1} \cap F_{2}\right)=J^{\prime}\left(F_{1}\right)+J^{\prime}\left(F_{2}\right)$, if $F_{1} \cap F_{2} \neq \varnothing$.

If the couple $\left(F_{1}, F_{2}\right)$ satisfies the (iii'), we say that $F_{1}$ and $F_{2}$ are $J^{\prime}$-independent, i.e. independent each other with respect to information $J^{\prime}$.

Also in fuzzy setting, we generalize the (iii'), setting

$$
J^{\prime}\left(F_{1} \cap F_{2}\right)=\Psi\left(J^{\prime}\left(F_{1}\right), J^{\prime}\left(F_{2}\right)\right) \text { if } F_{1} \cap F_{2} \neq \varnothing .
$$

The properties of the intersection between fuzzy sets are the similar to the $\left[\left(p_{1}\right)-\left(p_{4}\right)\right]$ [5] [6]. Therefore, we are looking for functions $(4)$ solutions of the system $\left[\left(P_{1}\right)-\left(P_{5}\right)\right]$. We have again the similar result:

Proposition 4.2 A class of solution of the system $\left[\left(P_{1}\right)-\left(P_{5}\right)\right]$ is

$$
\Psi_{k}(x, y)=k^{-1}(k(x)+k(y)) \text {, }
$$

where $k$ is any continuous, strictly increasing function $k:[0,+\infty] \rightarrow[0,+\infty]$ with $k(0)=0$ and $k(+\infty)=+\infty$.

From (5), we get

Proposition 4.3 A generalization of the $J^{\prime}$-independence property between two fuzzy set is

$$
J^{\prime}\left(F_{1} \cap F_{2}\right)=k^{-1}\left(k\left(J^{\prime}\left(F_{1}\right)\right)+k\left(J^{\prime}\left(F_{2}\right)\right)\right), \forall F_{1}, F_{2} \in F, F_{1} \cap F_{2} \neq \varnothing,
$$

where $k$ is any continuous, strictly increasing function $k:[0,+\infty] \rightarrow[0,+\infty]$ with $k(0)=0$ and $k(+\infty)=+\infty$.

Proof. The proof is similar to that given for crisp sets.

Remark. When $k$ is linear, the generalization (6) coincide with the property (iii').

\section{Conclusions}

In this paper we have proposed a genralization of $J$-independence property between crisp sets:

$$
J\left(C_{1} \cap C_{2}\right)=h^{-1}\left(h\left(J\left(C_{1}\right)\right)+h\left(J\left(C_{2}\right)\right)\right), \forall C_{1}, C_{2} \in C, C_{1} \cap C_{2} \neq \varnothing,
$$

where $h$ is any continuous, strictly increasing function $h:[0,+\infty] \rightarrow[0,+\infty]$ with $h(0)=0$ and $h(+\infty)=+\infty$.

Therefore, we have extended the result to fuzzy setting:

$$
J^{\prime}\left(F_{1} \cap F_{2}\right)=k^{-1}\left(k\left(J^{\prime}\left(F_{1}\right)\right)+k\left(J^{\prime}\left(F_{2}\right)\right)\right), \forall F_{1}, F_{2} \in F, F_{1} \cap F_{2} \neq \varnothing,
$$


where $k$ is any continuous, strictly increasing function $k:[0,+\infty] \rightarrow[0,+\infty]$ with $k(0)=0$ and $k(+\infty)=+\infty$.

\section{References}

[1] Kampé de Fériet, J. and Forte, B. (1967) Information et Probabilité. Comptes Rendus de l'Académie des Sciences Paris, 265, 110-114, 142-146, 350-353.

[2] Forte, B. (1969) Measures of Information: The General Axiomatic Theory. RAIRO Informatique Théorique et Applications, 63-90.

[3] Kampé de Feriét, J. (1970) Mesures de l'information fornie par un evénement. Colloque International du Centre National de la Recherche Scientifique, 186, 191-221.

[4] Benvenuti, P., Vivona, D. and Divari, M. (1990) A General Information for Fuzzy Sets. Uncertainty in Knowledge Bases, Lectures Notes in Computer Sciences, 521, 307-316. http://dx.doi.org/10.1007/BFb0028117

[5] Zadeh, L.A. (1965) Fuzzy Sets. Information and Control, 8, 338-353. http://dx.doi.org/10.1016/S0019-9958(65)90241-X

[6] Klir, G.J. and Folger, T.A. (1988) Fuzzy Sets, Uncertainty and Information. Prentice-Hall International Editions, Englewood Cliffs.

[7] Halmos, P.R. (1965) Measure Theory. Van Nostrand Company, Princeton.

[8] Benvenuti, P. (2004) L’opera scientifica. Roma, Ed.Univ.La Sapienza, Italia.

[9] Aczél, J. (1966) Lectures on Functional Equations and Their Applications. Academic Press, New York.

[10] Forte, B. (1970) Functional Equations in Generalized Information Theory. In: Applications of Functional Equations and Inequalities to Information Theory, Ed. Cremonese, Roma-Italy, 113-140.

[11] Ling, C.-H. (1995) Representation of Associative Functions. Publicationes Mathematicae, 12, 189-212. 